serum uric acid in different social classes, found that executives had a higher mean level than craftsmen. Fortythree per cent. of the executives, but only $12 \%$ of the craftsmen, had a level above $6.0 \mathrm{mg}$. per $100 \mathrm{ml}$. Distribution curves for executives and for medical students tended to be bimodal, with a second peak at $6.0 \mathrm{mg}$. per $100 \mathrm{ml}$; this was not seen in craftsmen and in high-school students, and its explanation is uncertain. No correlation was found in medical students between serum uric acid and aptitude, but in high-school students the serum uric acid was related to the number of extracurricular activities. There was no relation between the serum uric acid and social class of the family of origin. Executives and craftsmen did not differ in regard to age, health, or the intake of drugs, but the effects of possible differences in diet, consumption of alcohol, or exercise were not studied. The authors considered that any such differences were probably not great enough to cause the observed variation in serum-uric-acid levels, but this remains a matter of opinion. In all groups there was an association between serum uric acid and obesity, though at any weight the executives still showed a higher level than the craftsmen.

Unfortunately the estimation of uric acid presents difficulties. None of the colorimetric methods is satisfactory, partly because uric acid may be precipitated together with the serum proteins, partly because of non-specific colour changes, and partly because of the necessity for absolutely standard conditions of $\mathrm{pH}$, temperature, and reaction time. Some of these errors are eliminated when manual methods are replaced by the Autoanalyzer. Uricase-spectrophotometric methods are more precise than colorimetric methods, but here again laborious standardization is necessary. The unsatisfactory position for clinicians was emphasized in a recent survey initiated by the Empire Rheumatism Council, ${ }^{10}$ in which dried sera from three donors were sent to 36 hospital laboratories in Great Britain. Different estimates of uric acid in the same three sera showed a wide range of results ( 2.4 to $4.2 ; 2.1$ to 4.6 ; and 3.8 to $7.7 \mathrm{mg}$. per $100 \mathrm{ml}$. in the three cases). The least inconsistent results were obtained by centres using colorimetric (Autoanalyzer) methods. A wider scatter was seen with both colorimetric (manual) and uricasespectrophotometric methods. The survey emphasizes the need for carefully standardized conditions in estimations of uric acid carried out in hospital laboratories.

\section{Measles and Measles Vaccination}

The natural behaviour of infectious diseases is always prone to perpetual change. Not only does the virulence of the micro-organisms fluctuate, but there are also the alterations (not always easily measurable) in social environment and human behaviour. Dr. D. C. Miller's large-scale study of the measles epidemic of 1963, at p. 75 of this week's Fournal, analyses the incidence of the serious complications of this disease, and will be a useful source of information on which to base a national policy of vaccination against measles.

Death from measles in Britain is now unusual. In 1961 the rate was as low as 2 per 10,000 notifications-a figure which was repeated in Miller's experience in 1963. Furthermore, in one-half of the deaths there was an accompanying chronic disease or disability which in the majority was described as a serious handicap. It is reasonable, therefore, to conclude that in the past 30 years mortality from measles has been reduced to virtually the lowest figure possible. But there are other features of an infection to consider besides its mortality, and Miller directs our attention to the complications, and enlarges the previous studies sponsored by the College of General Practitioners. Broadly speaking, the complications in measles fall into two categories. Firstly, the virus itself produces not only local damage to the cells primarily involved (mainly in the respiratory and gastrointestinal tracts), but it may travel in the blood-stream to sites such as the central nervous system to produce encephalitis. It may well be that in those children who still die from respiratory complications (and in the 1961 epidemic these accounted for two-thirds of the deaths) the measles virus is the main factor, for in many the pneumonia was an early feature which occurred before or coincidentally with the rash. But the second, and much larger, group of complications is due to bacterial pathogens normally present in the upper respiratory tract which invade the areas of local cellular destruction. In this second group antibiotics-which as yet have no effect upon the viral component of the infectionhave made a considerable contribution to therapy.

Respiratory complications, therefore, bulk largest in the present series, producing, with otitis media, a rate of 63 per 1,000 cases. It is a surprising feature that, apart from the expected high rate in the first year of life, the incidence of respiratory complications showed little variation with age thereafter. This might be explained by the classification of severe bronchitis and pneumonia together and the predominance of bronchitis in the older age groups. Respiratory catarrh is a normal feature of measles, and though this is always alarming in the infant it seldom gives rise to concern after the age of 2 or 3 years.

Neurological complications were observed in 4 per 1,000 cases of measles, with encephalitis comprising about onequarter. Though the rate was very low up to the age of 9 years it was between 4 and 5 per 1,000 over this age. This resembles the greater susceptibility of the older child to encephalitis after primary smallpox vaccination, and suggests that some factor other than the virus is involved.

Does this present survey of measles in England and Wales add up to a strong argument for mass vaccination as Miller argues ? Into the balance must go a number of other factors. With a live, attenuated-virus vaccine ${ }^{12}$ it seems possible that lasting immunity will be achieved only by some degree of illness in the inoculated child. This may be reduced by gamma-globulin, but then a nice balance has to be made between the two components to avoid the "passive" acting as too effective a blanket for the " active." How frequently is the doctor going to be called to see the vaccinated child when it develops a more severe reaction? And are we to classify fits and convulsions occurring during an attack of measles as "neurological complications," whereas if they occur after live measles vaccine they are a "consequence of high fever"?

A killed virus vaccine might, of course, be added to the present quadruple cocktail (immunization against diphtheria, tetanus, whooping-cough, and poliomyelitis), but even with three injections it seems unlikely that permanent immunity would result. Moreover, it would be tragic if its action was merely to postpone an attack of measles into the age-group when complications such as encephalitis would be more

\footnotetext{
- Brit. med. F., 1960, 2, 368.

I Hendrickes, R. G., Montefiore, D., Sherman, P. M., and van der Wall, H. M., ibid., 1964, 1, 470 .
} 
common. To be really effective in preventing respiratory complications vaccination would need to be started very early - presumably about the age of 6 months. Have we any grounds for believing that this would be accepted on the scale necessary to affect the total figures significantly ? Miller paints an arresting picture of what he terms the "formidable toll exacted by a measles epidemic." On the other hand, the development of respiratory complications, for example, does not depend only on age-nutrition and social environment are also important. Overcrowded housing conditions and large families increase the incidence ; town dwellers have a higher incidence than rural inhabitants ; winter epidemics produce a higher rate of respiratory-tract infections than when the epidemic occurs in summer. It is likely that vaccination would be actively sought by those of higher social statusin whom respiratory complications, at any rate, would be less frequent-and be overlooked by those in less favourable circumstances, where the present rates would continue. Moreover, an incidence of complications of 67 cases out of 1,000 patients means that no fewer than 933 progressed to a straightforward recovery, and it might be suggested that the vaccination (with its attendant side-effects) of 10,000 persons in the hope of avoiding some 700 complications-nearly all susceptible to effective treatment-is a rather costly undertaking. These considerations do not necessarily apply to other populations, such as those in Nigeria and South America, where measles is severe and an important cause of death in childhood. In these countries there is an urgent need for effective protection against the disease, and $R$. G. Hendrickse and his colleagues ${ }^{3}$ in Nigeria found that a highly attenuated vaccine was effective, easy to administer, and acceptable.

René Dubos concludes his provocative essay on "Mirage of Health" with the words, "To grow in the midst of dangers is the fate of the human race," and we would do well to remind ourselves that artificial immunization is not an indefinitely extendible exercise. We have done well to rid ourselves of the captains of the men of death-some by vaccination, some by simpler measures of environmental hygiene. In Great Britain at the moment it is not necessarily logical to say, "We can produce a vaccine ; let us therefore use it."

\section{New Ideas on Carcinogenesis}

The current issue of the British Medical Bulletin, entitled "Mechanisms of Carcinogenesis : Chemical, Physical, and Viral," is a valuable document. As well as summarizing advances made during the past five years it gives a contemporary record of the thoughts and views of a group of experts. In 1947 and 1958 much the same ground was covered in issues of the Bulletin, and it is interesting, as Professor A. Haddow points out in his introduction to the present number, to see how emphasis has changed through the years.

Since 1947 advances in methods of measurement have changed the face of chemical and biochemical research. In particular radioisotopes, methods of measuring small amounts of radiation, chromatographic techniques, and advances in the field of spectrophotometry have made possible studies which could not even have been entertained 17 years ago. At the same time the development of high-speed centrifuges and of techniques for growing cells and even whole organs in vitro has made it possible to ask entirely new questions about the mechanism of carcinogenesis, and has revolutionized virological research in relation to cancer. Increasing use is being made of genetically defined and homogeneous animals and of animals free from specified pathogens, with the result that fewer uncontrolled variables interfere with the response of animals to particular forms of treatment.

The discovery of the role of the thymus ${ }^{1}$ in the immunological defence system has had wide repercussions in many fields, not least in the study of mechanisms of carcinogenesis. On this subject Sir Macfarlane Burnet writes in the Bulletin: "It may be that the most important effect of immunological processes is to be seen in the shape of the curve of age incidence of malignant disease. The peak is in early childhood, which would correspond to malignant disease initiated in the perinatal period of immunological inadequacy, and the accelerating rise with age after middle life may be in part associated with the progressive loss of capacity to initiate new immunological reactions with advanced age." According to $M$. C. Berenbaum the correlation between carcinogenicity and the ability to inhibit immune processes is imperfect, but close enough to suggest that the two effects are related. The inhibition of production of antibody by certain chemotherapeutic agents behoves caution in their use in the treatment of cancer, but at the same time may make it possible to replace cancerous organs by transplants of whole organs.

One of the difficulties facing those who try to associate chemical structure with a type of biological activity such as carcinogenicity is that substances may be changed by metabolic processes within the body. Thus, an apparent carcinogen may itself be inactive, whereas one of its metabolites is the proximal carcinogen. This is almost certainly so in the case of the aromatic amines and may be true too of the polycyclic hydrocarbons. Some of the biological alkylating agents may induce cancer directly, but the initial hope that, because of their simple chemical structure, studies on them would lead to an elucidation of a clear mechanism of carcinogenesis has not been fulfilled. Two new types of carcinogen, nitrosamines and lactones, have been discovered. The nitrosamines are themselves capable of alkylation, but whether this is the only mechanism by which they induce cancer is not certain. According to F. Dickens, one group of the carcinogenic lactones are carcinogenic by virtue of their alkylating activity but a second group may have an entirely different mode of action. The detection of increasing numbers of cancer hazards in the natural environment and the fact that some widely used pharmaceutical preparations such as iron-dextran, ${ }^{23}$ griseofulvin, and isonicotinic acid hydrazide have been shown to induce cancer in experimental animals provide food for thought. In particular, legislators are going to have to move away from the standpoint of not accepting known or potential cancer hazards at any level of risk, and instead arrive at compromise decisions on the basis of "the least evil" after all available evidence has been weighed.

Undoubtedly we are at the beginning of a period of intensive research on cancer viruses and on their relationship

\footnotetext{
1 See Brit. med. F., 1963, 1, 625.

2 Ibid., 1964, 1, 1583

3 Haddow, A., Roe, F. J. C., and Mitchley, B. C. V., ibid., 1964, 1, 1593.

4 Berwald, Y., and Sachs, L., Nature (Lond.), 1963, 200, 1182

${ }^{5}$ Brit. med. F., 1964, 1, 1197.

- Bell, T. M., Massie, A., Ross, M. G. R., and Williams, M. C., ibid., 1964, 1,1212 .
} 